\title{
Cycle-time and residence-time density approximations in a stochastic model for circulatory transport
}

\author{
Charles E. Smith ${ }^{1}$, Petr Lánský ${ }^{2}$ Te-Hsin Lung ${ }^{1}$, \\ ${ }^{1}$ Department of Statistics, North Carolina State University, Raleigh, NC 27695-8203, U.S.A. \\ ${ }^{2}$ Institute of Physiology and Center for Theoretical Study, Academy of Sciences of the Czech Republic, \\ Videñská 1083, 14220 Prague 4, Czech Republic
}

Address for correspondence:

Prof. Charles E. Smith

Department of Statistics

Biomathematics Graduate Program

North Carolina State University

Raleigh, NC 27695-8203

U.S.A.

$\mathrm{FAX}+919-515-7591$

e-mail cesmith@stat.ncsu.edu 
The concentration of a drug in the circulatory system is studied under two different elimination strategies. The first strategy, geometric elimination, is the classical one which assumes a constant elimination rate per cycle. The second strategy, Poisson elimination, assumes that the elimination rate changes during the process of elimination. The problem studied here is to find a relationship between the residence-time distribution and the cycle-time distribution for a given rule of elimination. While the presented model gives this relationship in terms of Laplace-Stieltjes transform, the aim here is to determine the shapes of the corresponding probability density functions. From experimental data, we expect positively skewed, gamma-like distributions for the residence time of the drug in the body. Also, as some elimination parameter in the model approaches a limit, the exponential distribution often arises. Therefore, we use Laguerre series expansions, which yield a parsimonious approximation of positively skewed probability densities that are close to a gamma distribution. The coefficients in the expansion are determined by the central moments, which can be obtained from experimental data or as a consequence of theoretical assumptions. The examples presented show that gamma-like densities arise for a diverse set of cycle-time distributions and under both elimination rules.

1. Introduction. The residence-time functions of drugs in the body, reflected by the concentration-time curves, form an experimental counterpart to theoretical studies on residence-time distributions. The most commonly employed approach to the problem of drug residence time is to characterize the body as a system of discrete compartments. The compartmental models are usually considered in their deterministic versions which can then be randomized. A different approach was initiated by Waterhouse and Keilson (1972) and later pursued by Weiss $(1982,1984,1986)$, for a review see Weiss (1991). It is basically a stochastic approach. In this case it is assumed that following a single, instantaneous injection of a bolus of a substance into the vascular system, the injected substance starts to circulate and its elimination takes place at a specific point. The circulation time and elimination are characterized as random variables. A comparison between compartmental, noncompartmental and circulatory models was recently made by (Mari, 1993; Ďurišová, 1995). 
Under this scenario for circulatory models, it is presumed that the residence time of the drug in the body is composed of several complete cycles and the additional time from the end of last complete cycle to elimination. In reality, this additional time may form a substantial part of the residence time, however it is neglected here. To include this residual time into the analyses can be done, but only by considering the specific conditions of the experiment, e.g., site of injection, cardiac output (Mari, 1995). On the other hand, the model studied here is in its basic form and can be applied after appropriate modifications to a variety of situations. In its basic form, the model has two, unknown (unspecified), components - distribution of the cycle time and the elimination rule.

Generally, the concentration-time curves represent the standard result of pharmacokinetic experiments. In the second part of this paper, a relation between the residence-time distribution and the concentration-time curve is invoked. This is the critical issue for relating the model to experimental results. Once the residence-time distribution is determined, we can try to deduce the cycle-time distribution and drug elimination mechanism, which are very difficult to measure directly.

The main goal of this paper is to relate cycle-time distributions to residence-time distributions which can be determined experimentally. We assume that the first four moments of the cycle-time are available and then use them to construct the residencetime density via a Laguerre series (L-series) approximation. The theoretical background needed for this purpose is summarized below and later illustrated on several examples, for more details about the method and its application see (e.g., Bowers, 1966; Beekman, 1974; Tiku et al., 1986; Smith, 1991).

2. Residence-time distribution and concentration-time curve. The residence time, $R$, of a drug molecule in a system is a non-negative random variable with a cumulative distribution function $H(t)$. Its differentiability ensures the existence of the corresponding probability density function, $h(t)=\mathrm{d} H / \mathrm{d} t$. The log-convexity of the probability density 
function $h(t)$ implies that that $C V \geq 1$, (CV is the coefficient of variation, $\mathrm{CV}=$ standard deviation/mean), (Weiss, 1986). Some properties of the random variable $R$ may be better represented by other functions, for example by the survival function, $\bar{H}(t)=1-H(t)$, or by the failure rate (intensity) function, also called fractional elimination rate, $k(t)=h(t) / \bar{H}(t)$ (probability of elimination at a given time under the condition that the molecule has not been eliminated up to this time). Discrimination between monotonically decreasing and increasing fractional elimination rates $k(t)$ provides a useful method for characterizing the residence-time distribution (Weiss, 1991). Further, for $k(t)$ being constant, at least asymptotically, the density $h(t)$ is asymptotically exponential. A special class of the distributions with nonincreasing $k(t)$ are so called completely monotone distributions; a function on $(0,+\infty)$ is completely monotone if and only if it is a mixture of exponentials (Feller, 1966). For a random variable with a completely monotone density it follows that the CV $\geq 1$ and as we will see below, the CV plays an important role in determining the shape of the distribution, particularly for the gamma distribution.

In order to relate the distribution of $R$ to observable quantities, we have to shift from an individual molecule to an ensemble of them. Commonly it is considered that

$$
h(t) \approx \frac{C(t)}{\int_{0}^{\infty} C(t) \mathrm{d} t}
$$

where $C(t)$ is the measured concentration-time curve, also known as the drug disposition rate and $\int_{0}^{\infty} C(t) \mathrm{d} t$ is often denoted by $A U C$ (area under the curve). In other words, $C(t)$ reflects the concentration at the output and must not be confused with concentration of the drug remaining in the system, although this latter quantity is in some studies the variable of interest, e.g., Durišová et al,, 1995. To avoid possible confusion, the definition of concentration-time curve must be precise and then there is no doubt as to the applicability of (2.1) (Lánský, 1996). Equation (2.1) directly implies the formulas for the moments of the residence time, 


$$
\mathrm{E}\left(R^{n}\right)=\int_{0}^{\infty} t^{n} h(t) \mathrm{d} t \approx \frac{\int_{0}^{\infty} t^{n} C(t) \mathrm{d} t}{\int_{0}^{\infty} C(t) \mathrm{d} t} .
$$

To estimate the mean residence time, or the distribution of the residence time, from the concentration-time curve is the first task in this type of experiment. When this is done, one may ask to what extent the estimated residence-time distribution fits a theoretical one derived from a mathematical model. In this manner, an inference about the cycle-time distribution and about the elimination rule is based on the concentrationtime curve. The distributions suitable for this purpose were discussed in detail by Weiss (1991).

3. Laguerre series representation of probability densities. Our method for residencetime distribution analysis is based on the L-series approximation (Bowers, 1966; Beekman, 1974) of a density by a linear combination of gamma densities $f(x ; \alpha, \beta)$ given by

$$
f(x ; \alpha, \beta)=\frac{x^{\alpha-1} e^{-x / \beta}}{\beta^{\alpha} \Gamma(\alpha)}
$$

where $\alpha>0$ and $\beta>0$ are the shape and scale parameters, respectively. For the general case, let us assume that the density $\bar{g}(x)$ of a random variable $Y$ is to be approximated. To simplify the notation we will study a scaled random variable $X=k Y$ with density $g(x)$ and the resultant density will be rescaled back after the procedure using the relationship, $\bar{g}(x)=k g(k x)$. The scaling aims to ensure that $\mathrm{E}(X)=\operatorname{Var}(X)=\alpha$ and this is achieved by taking $k=\alpha / \mathrm{E}[Y]=\mathrm{E}[Y] / \operatorname{Var}[Y]$. The scaling permits us to evaluate the density $g(x)$ in terms of gamma densities (3.1) with $\beta=1$. We use the notation, $f(x ; \alpha)=f(x ; \alpha, 1)$. Note that $\alpha^{-1}=\mathrm{CV}^{2}(X)=\mathrm{CV}^{2}(Y)$ and we can use these relations for estimating $k$ from experimental samples by $\hat{\alpha} / \bar{y}$, where $\hat{\alpha}$ is the estimate of $\alpha$ and $\bar{y}$ is the sample mean of $Y$; also $\widehat{k}$ is an estimate of $\beta^{-1}$ in (3.1). The series expansion for the 
density $g(x)$ of the random variable $X$ is

$$
g(x)=\frac{x^{\alpha-1} e^{-x}}{\Gamma(\alpha)}\left\{1+A_{3} L_{3}^{(\alpha)}(x)+A_{4} L_{4}^{(\alpha)}(x)+\ldots\right\}
$$

where $L_{i}^{(\alpha)}(x)$ is the $i$-the order Laguerre polynomial. The density $g(x)$ may be used to represent either cycle or residence time depending on the context, however, in this paper we consider only the case of the residence-time density approximation. In the approximation we will only use moments up to 4 th order. The constants $A_{3}, A_{4}$ can be evaluated from the third and fourth moments of $X$, and by using the 3rd and 4th Laguerre polynomials, we finally obtain

$$
\begin{aligned}
& g(x) \approx f(x ; \alpha)[1-A+B]+f(x ; \alpha+1)[3 A-4 B]+f(x ; \alpha+2)[6 B-3 A] \\
& +f(x ; \alpha+3)[A-4 B]+f(x ; \alpha+4) B
\end{aligned}
$$

where $A=\left(\mu_{3}-2 \alpha\right) / 6, B=\left(\mu_{4}-12 \mu_{3}-3 \alpha^{2}+18 \alpha\right) / 24$ with $\mu_{n}$ being the $n$th central moment of $X$. Taking $A$ and $B$ equal zero in (3.3) we fit the density $g(x)$ to a gamma density by the method of moments, which means that first two moments of $X$ are the same as the first two moments of $f(x ; \alpha)$. Using the complete formula ensures that the first four moments of $X$ are the same as those computed from the right hand side of (3.3). An illustration of the role of the constants $A$ and $B$ is given in Fig. 1 for $\alpha=1.5$. When $A$ and $B$ are of the same sign then the approximating curve is not very different from the true one, whereas for $A$ and $B$ of the opposite sign we can see substantial changes in both shape and in mode location. The possible range of shape changes caused by variations in $A$ and $B$ is much broader than depicted in the figure and it also strongly depends on the value of parameter $\alpha$. For example, for a fixed value of $A$ and $B$, the deviation from a gamma becomes less pronounced as $\alpha$ increases.

4. The model. For the model we consider the residence time, $R$, to be composed of a 
random number of cycle times, $T_{i}$. The general form of the model is

$$
\begin{gathered}
R=\sum_{i=1}^{J} T_{i}+T_{0} \quad \text { if } J>0 \\
R=T_{0} \text { if } J=0
\end{gathered}
$$

where $J$ is a discrete random variable, $\operatorname{Prob}(J=j)=p_{j}$. The model (4.1) is still too complicated for our present effort, so we further assume that $\operatorname{Prob}\left(T_{0}=0\right)=1$, (i.e., the particle is eliminated at the instant of completing the cycle) and $\operatorname{Prob}(J=0)=0$ (i.e., the first cycle is obligatory for all the injected molecules). This is a standard approach (Weiss, 1982) and actually it gives an upper boundary for the residence time. Further, we assume that the $T_{i}$ 's are independent and identically distributed random variables with a common cumulative distribution function, $G(t)$, i.e. $G_{i}(t)=G(t)$ for any $i$, and jth moment denoted by $\mathrm{E}\left(T_{i}^{j}\right)=\mathrm{E}\left(T^{j}\right)$ for any $i$. This assumption is biologically plausible and makes the model more tractable. The number of discrete distributions suitable for the number of cycles seems to be limited, in fact only the geometric has been used previously. Nevertheless, under the assumptions of the model, we may specify an elimination scheme, or equivalently the distribution of $J$. Let us denote by $P(s)$ the probability generating function of $J, P(s)=\sum_{j=0}^{\infty} p_{j} s^{j}$. Then, for the Laplace-Stieltjes (LS) transform of $H(t)$, denoted by $H(s)$, we obtain from (4.1) recalling the assumption $\operatorname{Prob}\left(T_{0}=0\right)=1$, that

$$
H(s)=P(G(s))
$$

where $G(s)$ is LS transform of the cycle time. This is our basic model and (4.2) can be used for evaluation of the residence-time density from the cycle-time density, or vice versa, either analytically or by using a numerical inversion of the LS transform. While this works theoretically, practically at the experimental level, it is not possible to 
determine the density, $h(t)$, with enough precision to use (4.2) directly. Therefore the evaluation of the density has to be done using the moments. Formula (4.2) provides us with the moments of the residence time via the moments of $J$ and $T$,

$$
\begin{gathered}
E(R)=\mathrm{E}(J) \mathrm{E}(T) \\
\mathrm{E}\left(R^{2}\right)=\mathrm{E}(J) \mathrm{E}\left(T^{2}\right)+\left(\mathrm{E}\left(J^{2}\right)-\mathrm{E}(J)\right) \mathrm{E}^{2}(T) \\
\mathrm{E}\left(R^{3}\right)=\mathrm{E}(J) \mathrm{E}\left(T^{3}\right)+3\left(\mathrm{E}\left(J^{2}\right)-\mathrm{E}(J)\right) \mathrm{E}\left(T^{2}\right) \mathrm{E}(T)+\left(\mathrm{E}\left(J^{3}\right)-3 \mathrm{E}\left(J^{2}\right)+2 \mathrm{E}(J)\right) \mathrm{E}^{3}(T) \\
\mathrm{E}\left(R^{4}\right)=\mathrm{E}(J) \mathrm{E}\left(T^{4}\right)+4\left(\mathrm{E}\left(J^{2}\right)-\mathrm{E}(J)\right) \mathrm{E}\left(T^{3}\right) \mathrm{E}(T)+3\left(\mathrm{E}\left(J^{2}\right)-\mathrm{E}(J)\right) \mathrm{E}^{2}\left(T^{2}\right) \\
+6\left(\mathrm{E}\left(J^{3}\right)-3 \mathrm{E}\left(J^{2}\right)+2 \mathrm{E}(J)\right) \mathrm{E}^{2}(T) \mathrm{E}\left(T^{2}\right)+\left(\mathrm{E}\left(J^{4}\right)-6 \mathrm{E}\left(J^{3}\right)+11 \mathrm{E}\left(J^{2}\right)-6 \mathrm{E}(J)\right) \mathrm{E}^{4}(T)
\end{gathered}
$$

We can see from these formulas that once the distributions of $J$ and $T$ are specified, we can use the approximation (3.3).

4.1. Geometric distribution of the number of cycles. Weiss (1984) used the model (4.1), assuming that the probability that a molecule of a drug is eliminated after a single passage through the circulation is $p(0<p<1)$ independent of the number of previous cycles. It is a linear model in the sense that the eliminated fraction of the substance is at any moment linearly proportional to the amount of drug within the system. It follows from this assumption that the probability of elimination at the $j$ th cycle has a geometric distribution, $\operatorname{Prob}(J=j)=p q^{j-1}$, for $j \geq 1$, where $q=1-p$. For this discrete distribution $P(s)=p s /(1-q s)$ and it follows from (4.2) that

$$
H(s)=\frac{p G(s)}{1-q G(s)} .
$$

Using (4.7) or directly using (4.3)-(4.6), we can compute the moments of the residence 
time, $R$, in terms of the moments of cycle time, $T$, and the probability of elimination, $p$, we have

$$
\begin{gathered}
\mathrm{E}(R)=\frac{1}{p} \mathrm{E}(T), \\
\mathrm{E}\left(R^{2}\right)=\frac{1}{p}\left(\mathrm{E}\left(T^{2}\right)+2 \frac{q}{p} \mathrm{E}^{2}(T)\right), \\
\mathrm{E}\left(R^{3}\right)=\frac{1}{p}\left(\mathrm{E}\left(T^{3}\right)+6 \frac{q}{p} \mathrm{E}\left(T^{2}\right) \mathrm{E}(T)+6\left(\frac{q}{p}\right)^{2} \mathrm{E}^{3}(T)\right), \\
\mathrm{E}\left(R^{4}\right)=\frac{1}{p}\left(\mathrm{E}\left(T^{4}\right)+8 \frac{q}{p} \mathrm{E}(T) \mathrm{E}\left(T^{3}\right)+36\left(\frac{q}{p}\right)^{2} \mathrm{E}\left(T^{2}\right) \mathrm{E}^{2}(T)+6 \frac{q}{p} \mathrm{E}^{2}\left(T^{2}\right)+24\left(\frac{q}{p}\right)^{3} \mathrm{E}^{4}(T)\right) .
\end{gathered}
$$

It follows from (4.7) that for an exponentially distributed cycle time, the residence time is also exponential, and vice versa. For many drugs, the probability of elimination within one cycle is very small, (van Rossum et al. (1989) suggest the value $p=1 / 100$ ), therefore the behavior of the residence-time distribution under the condition $p \rightarrow 0_{+}$is of interest. In the limit, of a decreasing probability of elimination, the residence time is exponentially distributed. This holds independently of the type of the cycle-time distribution (Lánský, 1996).

3.2. Poisson distribution of the number of cycles. The assumption that the number of cycles is governed by the geometric distribution implicitly states that the probability of entering higher numbers of cycles is monotonically decreasing. In other words it says that the amount of drug eliminated throughout the experiment is a monotonically decreasing function of time. However, a different time course of elimination is also plausible, for example one that slowly increases and achieves its maximum after some time delay, perhaps due to a reversible binding of the drug. The Poisson distribution for the number of cycles until elimination would be a suitable model for such a situation. This strategy produces a nonlinear relationship between the amount of substance which 
is eliminated at a given moment and the total amount remaining within the system at this moment (reflected by its concentration) is variable. The elimination rule considered here does not allow elimination during the first cycle, so $J$ has to take values in the set $\{1,2, \ldots\}$. Therefore, let us, consider $J$ with the probability generating function $P(s)=s \exp (\nu(s-1))$, where $\nu>0$ is a parameter, which is a shifted Poisson distribution. The LS of the residence-time distribution becomes

$$
H(s)=G(s) \exp \{\nu(G(s)-1)\}
$$

The mean residence time is, as intuitively expected since $\mathrm{E}(J)=\nu+1$,

$$
\mathrm{E}(R)=(1+\nu) \mathrm{E}(T)
$$

This relation shows that the amount of overestimation of the residence time by making the first cycle obligatory is at most $\mathrm{E}(T)$. Further,

$$
\begin{aligned}
\mathrm{E}\left(R^{2}\right) & =(1+\nu) \mathrm{E}\left(T^{2}\right)+(2+\nu) \nu \mathrm{E}^{2}(T) \\
\mathrm{E}\left(R^{3}\right) & =(1+\nu) \mathrm{E}\left(T^{3}\right)+3 \nu(2+\nu) \mathrm{E}\left(T^{2}\right) \mathrm{E}(T)+\nu^{2}(3+\nu) \mathrm{E}^{3}(T) \\
\mathrm{E}\left(R^{4}\right) & =(1+\nu) \mathrm{E}\left(T^{4}\right)+(2+\nu) 4 \nu \mathrm{E}\left(T^{3}\right) \mathrm{E}(T)+ \\
& +3 \nu \mathrm{E}\left(T^{2}\right)\left((2+\nu) \mathrm{E}\left(T^{2}\right)+2 \nu(3+\nu) \mathrm{E}^{2}(T)\right)+(4+\nu) \nu^{3} \mathrm{E}^{4}(T)
\end{aligned}
$$

5. Examples. The relations for the moments derived in the previous two sections are straightforward and can be used to compute the moments of the residence time from the moments of the cycle time. On the other hand, to compute the residence-time density analytically from the cycle-time density is possible only in a few special cases. For other cases, alternative methods such as simulations, numerical solutions or 
approximations have to be used. We will use the L-series approximation described in Section 3 to evaluate the residence-time density from its moments as a function of the cycle-time moments that were computed in the previous section. In (3.3) we used the third and fourth central moments $\mu_{n}$ and these are related to the moments about the origin by $\mu_{3}=\mathrm{E}\left(X^{3}\right)-3 \mathrm{E}(X) \mathrm{E}\left(X^{2}\right)+2 \mathrm{E}^{3}(X)$ and $\mu_{4}=\mathrm{E}\left(X^{4}\right)-4 \mathrm{E}(X) \mathrm{E}\left(X^{3}\right)+$ $6 \mathrm{E}^{2}(X) \mathrm{E}\left(X^{2}\right)-3 \mathrm{E}^{4}(X)$. The shape of the density function has often been characterized by its higher moments (Johnson and Kotz, 1970). Two commonly used moment plots are: (1) A Pearson plot in which the coefficient of excess $\beta_{2}-3$ is plotted vs. skewness $\beta_{1}$, where $\beta_{1}=\mu_{3}^{2} / \mu_{2}^{3}$ and $\beta_{2}=\mu_{4} / \mu_{2}^{2}$, (2) A plot of $\sqrt{\beta_{1}}$ vs. CV. These plots characterize the similarity of the given distribution to a gamma distribution, since the gamma distribution produces the line $\beta_{2}-3=3 \beta_{1} / 2$, and the line $\sqrt{\beta_{1}}=2 \mathrm{CV}$, which will be used in figures 5 and 6 .

5.1. Exponentially distributed cycle time. For this cycle time distribution we can derive analytical expressions for the residence-time densities and in this way illustrate the accuracy and the utility of our method. The exponential density is described by (3.1) with $\alpha=1$ and with scale parameter, $\beta$, solely characterizing the distribution. Then for the geometric elimination model (4.7), the residence time is exponentially distributed with scale parameter $\beta / p$. This means that the fit by a single gamma density (as mentioned above, the exponential is a special case with $\alpha=1$ ) is exact and no additional improvement can be achieved from the L-series method. For the Poisson elimination model (4.12), the exponential distribution of the cycle time does not produce an exponential residence time; instead when the cycle time is exponentially distributed with the scale parameter $\beta$, the density of the residence time becomes

$$
h(t)=\beta^{-1} \exp (-\nu-t / \beta) I_{0}(2 \sqrt{\nu t / \beta})
$$

where $I_{0}$ is a modified Bessel function of order 0 (Lánský, 1996). Figure 2 shows the 
theoretical densities $h(t)$ given by (5.1) and their L-series approximations $g(t)$ obtained by using (3.3). It is clear from the values of $A$ and $B$ that the corresponding gamma densities $f(t ; \alpha, \beta)$ given by (3.1) are close to the L-series approximation and consequently to the theoretical residence-time densities. Recall that if we had set $A=B=0$ in (3.3), the corresponding gamma densities in Fig. 2. would have the parameters ensuring that their first two moments are identical with the first two moments of the density (5.1). We can deduce from the picture that in all three cases: (1) the gamma density is fairly close to the exact shape of the residence-time density, (2) the L-series approximation improves the fit only slightly, since the gamma density is already very close to the exact one.

5.2. Gamma distributions for cycle time. A natural extension is to consider a gamma density, rather than its special case - the exponential, for the cycle time. For the gamma density (3.1), the shapes can be divided into two separate groups with respect to the value of the parameter $\alpha$. For $\alpha \in(0,1)$ it follows that $C V>1$, and this distribution has played an important role in pharmacokinetics (Wise, 1985). For $\alpha>1$, the $\mathrm{CV}<1$ and the distribution has a unimodal shape. For both cases, $\mathrm{E}(X)=\alpha \beta$, $\operatorname{Var}(X)=\alpha \beta^{2}$ and $\mathrm{CV}^{2}=1 / \alpha$ and higher moments are $\mathrm{E}\left(X^{r}\right)=\beta^{r} \Gamma(\alpha+r) / \Gamma(\alpha)$ (Johnson and Kotz, 1970). To avoid possible confusion, the parameters in this subsections are further indexed by $C$ to denote parameters of cycle time. For a gamma cycle-time distribution, the residence-time density in the geometric elimination model (4.7) is exactly a gamma in the hypothetical case when $p=1$ (the residence time coincides with the cycle time) and when $p \rightarrow 0_{+}$(the exponential limit of the residencetime distribution in the case of a small elimination rate). For other values of $p$, the moment plots were close to the gamma line and the L-series approximation was dominated by the leading gamma term as can be seen from the expressions for $A$ and $B$ of the L-series (3.3) in terms of the parameter of the cycle time $\alpha_{C}$ and elimination parameter $p$ 


$$
\begin{gathered}
-12- \\
A=\frac{\alpha_{C}^{2}\left(\alpha_{C}-1\right) p q}{6\left(p+\alpha_{C} q\right)^{3}} \\
B=\frac{\alpha_{C}^{2} p\left(5 p q+6 \alpha_{C} q\left(\alpha_{C}-1\right)+\alpha_{C} p q\left(7 \alpha_{C}-12\right)\right)}{24\left(p+\alpha_{C} q\right)^{4}} .
\end{gathered}
$$

The Erlang distribution, is a special case of (3.1) where $\alpha_{C}$ is replaced by $n \in N$. In this case substituting into (4.7) and taking the inverse LS transform we get the density $h(t)$ of the residence time in the form of a mixture of exponentials. For example, in the case $n=2$, then

$$
h(t)=\frac{p}{2 \beta_{C} \sqrt{q}}\left[\exp \left(-(1-\sqrt{q}) t / \beta_{C}\right)-\exp \left(-(1+\sqrt{q}) t / \beta_{C}\right)\right]
$$

$\mathrm{E}(R)=2 \beta_{C} / p, \operatorname{Var}(R)=2 \beta_{C}^{2}(1+q) / p^{2}$ and $C^{2}(R)=(1+q) / 2$. This is not illustrated but gives a further test of the accuracy of the L-series approximation. More importantly, it provides a mechanism for generating a mixture of exponentials residence-time distribution, which has been used previously (Wise and Borsboom, 1989; Mari, 1993; Ďurišová, 1995).

A further example is obtained by assuming a gamma distribution of the cycle time under the Poission elimination scheme. Also here an artificial limiting case exists, namely, for $\nu=0$ the gamma density of the cycle time coincides with the gamma density for the residence time. Further, substituting the LS transform of the gamma distribution (3.1), $G(s)=\left(s \beta_{C}+1\right)^{-\alpha_{C}}$, into (4.12) and taking $\nu \rightarrow+\infty$ and simultaneously $\alpha_{C} \rightarrow 0+$ (a high number of very short cycles is needed for elimination) in such a way that $\alpha_{C} \nu \rightarrow \psi$ then (4.12) takes the form

$$
H(s)=\left(\frac{1}{s \beta_{C}+1}\right)^{\psi}
$$

We can see that the residence time is asymptotically gamma distributed with the same 
scale parameter $\beta_{C}$ as the cycle time, but with a new shape parameter $\psi$. This is illustrated in Fig. 3, where with increasing $\nu$ and decreasing $\alpha_{C}\left(\nu \alpha_{C}=1\right)$ an exponential distribution for the residence time is rapidly approached even for relatively small values of $\nu$.

5.3. Inverse Gaussian distribution for cycle time. A more stringent test for our Lseries method is provided by the inverse Gaussian (IG) distribution. This distribution has a physical interpretation and can be derived as the first-passage-time distribution of the Wiener process with drift $\mu$ and infinitesimal variance $\sigma^{2}$ across a boundary at a distance $D$ from the origin. The density function is

$$
g(x)=\frac{D}{\sigma \sqrt{2 \pi x^{3}}} \exp \left\{-\frac{(D-\mu x)^{2}}{2 \sigma^{2} x}\right\} .
$$

The assumption $\mu>0$ ensures that the mean of the distribution (5.6) is finite. The most common form of (5.6) in the statistical literature uses the parameters $a=D / \mu$, $b=D^{2} / \sigma^{2}$ which produces

$$
g(x)=\sqrt{\frac{b}{2 \pi x^{3}}} \exp \left\{-\frac{b(x-a)^{2}}{2 a^{2} x}\right\}
$$

for a review of the properties of this distribution (5.6), resp. (5.7), see Chhikkara and Folks (1989). The LS transform of (5.6) is

$$
G(s)=\exp \left\{\left(\frac{D \mu}{\sigma^{2}}\right)\left(1-\sqrt{1+\frac{2 \sigma^{2} s}{\mu^{2}}}\right)\right\}=\exp \left\{\frac{b}{a}\left(1-\sqrt{1+\frac{2 a^{2} s}{b}}\right)\right\}
$$

and it can be substituted into the formula for $H(s)$. The mean, variance and square of the coefficient of variation for distribution (5.6), resp. (5.7), are $\mathrm{E}(X)=D / \mu=a$, $\operatorname{Var}(X)=D \sigma^{2} / \mu^{3}=a^{3} / b, \mathrm{CV}^{2}=\sigma^{2} / \mu D=a / b$ and higher moments can be computed by using (5.8). The IG distribution produces the line $\beta_{2}-3=5 \beta_{1} / 3$, and the line $\sqrt{\beta_{1}}$ 
$=3 \mathrm{CV}$ in the moment plots, which will be used in figures 5 and 6. Sheppard (1971) and Weiss (1982) used the IG distribution (5.6) for the cycle time in circulatory models.

The L-series approximation under the assumption that the cycle time $T$ has an IG distribution is illustrated in Fig. 4. The results, under the geometric elimination rule, are shown in Fig. 4a. as a function of the elimination parameter $p$. When $p=1$, an artificial case, the residence-time and the cycle-time distributions are identical. The Lseries approximates this case fairly well and shows that the method is useful for nonGamma cycle-time distributions as well. As $p$ decreases, the residence-time density becomes less peaked and more spread out, however the position of the mode changes only slightly. The approach to the theoretical exponential limit $\left(p \rightarrow 0_{+}\right)$is apparent even when $p=0.1$ (lower curve). The corresponding plots, when the parameter $a$ of the IG distribution becomes larger, with $b$ fixed, indicate that the L-series approximation for $p$ close to 1 is not as accurate as in Fig. 4a., but the approach to the exponential limit is still quite rapid. This point is reiterated in the discussion of moment plots.

Similarly in Fig. 4b., the L-series approximation to the residence-time density with the Poisson elimination rule are presented. The values of the parameter $\nu$ are chosen to correspond to those of $p$ in Fig. 4a. in such a way that the mean number of cycles until elimination are the same in both cases, i.e., $\nu=1 / p-1$. For small $\nu$ ( $p$ close to 1$)$, this figure is similar to Fig. 4a., however as $\nu$ becomes larger ( $p$ close to 0 ) the differences are quite apparent. Here the position of the mode moves rapidly to the right and the distribution becomes more similar to a Gaussian than to an exponential.

In Fig. 5a. we have a plot of the skewness, $\sqrt{\beta_{1}}$, vs $C V$ for the residence times with geometric elimination and with the cycle time $T$ having an IG distribution. The expressions for the $\mathrm{CV}$ and the $\sqrt{\beta_{1}}$ measure of skewness are

$$
\mathrm{CV}=\sqrt{\frac{b q+a p}{b}}
$$




$$
\sqrt{\beta_{1}}=\frac{3 a p(b q+a p)+b^{2} q(2+p)}{\sqrt{b}(b q+a p)^{3 / 2}} .
$$

The straight lines in the plot represent the relationship for a gamma distribution (lower line) and IG (upper line). Four different sets of parameters, $b=1.0$ and $a=0.25,0.64$, 1.44 and 2.25 , corresponding to cycle time CV's of $0.5,0.8,1.2$ and 1.5 for the IG are considered. The four curves all start on the IG line, corresponding to $p=1$, and with decreasing $p$ approach the exponential limit, the point with coordinates $(1,2)$ on the gamma line. While the upper three curves are quadratic-like, the lower curve, corresponding to Fig. 4a., shows that the approach from the IG line need not be monotonic.

Figure 5b. shows the corresponding plot for the residence times with Poisson elimination. The expressions for the CV and the $\sqrt{\beta_{1}}$ measure of skewness now are

$$
\begin{gathered}
\mathrm{CV}=\sqrt{\frac{b \nu+a(1+\nu)}{b(1+\nu)^{2}}} \\
\sqrt{\beta_{1}}=\frac{b^{2} \nu+3 a(b \nu+a(1+\nu))}{\sqrt{b}(b \nu+a(1+\nu))^{3 / 2}} .
\end{gathered}
$$

Note that the starting point $(\nu=0)$ of each curve on the IG line is the same as in Fig. 5a. As $\nu$ increases, the curves do not approach an exponential limit, but instead decrease toward the origin in close proximity to the gamma line. This behaviour is depicted in a different way in Fig. 6., where the excess, $\beta_{2}-3$, vs the square of the above measure of skewness, $\beta_{1}$, is plotted for cycle time CV's of $0.8,0.7,0.6$ and 0.5 with

$$
\beta_{2}=\frac{15 a^{3}(1+\nu)+b^{3} \nu(1+3 \nu)+6 a b^{2} \nu(2+\nu)+3 a^{2} b\left(1+\nu^{2}+7 \nu\right)}{b(b \nu+a(1+\nu))^{2}}
$$

Note again the proximity of the curves to the gamma distribution line for large $\nu$. For small $\nu$ and a small cycle-time CV, the curve actually starts in the opposite direction to 
its asymptotic limit. The proximity to the gamma line can also be seen by calculating the values of the L-series coefficients $A$ and $B$, analogously to (5.2) and (5.3).

The corresponding plot under geometric elimination is not shown, but approaches the exponential limit point in an almost monotonic manner, as can be seen from the above expression for $\sqrt{\beta_{1}}$ and from

$$
\beta_{2}=\frac{9 b^{3}(q-p)+3 b p^{2} a^{2}(5 q+1)+6 a b^{2} p q(p+3)+15 a^{3} p^{3}+b^{3} p^{2}(10-p)}{b(b q+a p)^{2}},
$$

or from the values of the L-series coefficients $A$ and $B$, analogously to (5.2) and (5.3).

6. Conclusions. (1) Assuming an elimination rule and a cycle-time distribution we are able to compute the moments of the residence time and based on them to approximate the residence-time density via the L-series method. Having the residence-time density available, the other characteristics such as the survival function or the fractional elimination rate can be easily evaluated. (2) The analytical results produce a wide variety of residence-time densities depending on which elimination rule and cycle time distribution are assumed. However, our numerical illustrations reveal that the resulting residence-time distributions are always relatively close to the gamma density and hence the L-series method is applicable. (3) The two most commonly used distributions for the residence time are the J-shaped gamma density and the mixture of exponentials (Wise, 1985; Wise and Borsboom, 1989). The initial assumptions leading to these distributions are quite different, thus theoretically the two models are distinguishable. An open question is whether these two distributions are also practically distinguishable from relatively sparse residence-time data. In many situations, it may be that only an expert can decide which underlying assumptions are more biologically plausible. (4) The approach presented in this paper also permits us to go in the opposite direction, namely to approximate the cycle-time density after assuming an elimination rule and taking the residence-time density from the experimental data. 


\section{Acknowledgments}

The authors acknowledge the support of the Office of Naval Research Grant N00014-90J-1646 and Grant Agency of the Czech Republic grant 309/95/0927. The second author is grateful to the Department of Statistics at North Carolina State University for their warm hospitality during his visit.

\section{REFERENCES}

Beekman, J.A. 1974. Two Stochastic Processes. Stockholm: Almqvist \& Wiksell.

Bowers, Jr. N.L. 1966. Expansion of probability density functions as a sum of gamma densities with applications in risk theory. Trans. Soc. Actuaries. 18, 125-137.

Chhikara, R. S. and J. L. Folks 1989. The Inverse Gaussian Distribution. New York: Dekker.

Ďurišová, M., L. Dedík and M. Balan 1995. Building a structured model of a complex pharmacokinetic system with time delays. Bull. Math. Biol. 57. 787-808.

Feller, W. 1966. An Introduction to Probability Theory and Its Applications. Vol. 2. New York: Wiley.

Johnson, N. L. and S. Kotz 1970. Continuous Univariate Distributions. Vol. 1. New York: Wiley.

Lánský, P. 1996. A stochastic model for circulat,ory transport in pharmacokinetics. Math. Biosci. (in press).

Mari, A. 1993. Circulatory models of intact-body kinetics and their relationship with compartmental and noncompartmental analysis. J. theor. Biol. 160, 509-531.

Mari, A. 1995. Determination of the single-pass impulse response of the body tissue with circulatory models. IEEE Trans. Biomed. Engn. 42, 304-312.

Rossum, J. M. van, J. G. M. de Bie, G. van Lingen and H. W. A. Teeuwen. 1989. Pharmacokinetics from a dynamical system point of view. J. Pharmacokin. Biopharmac. 17, 365-397. 
Sheppard, C. W. 1971. Stochastic models for tracer experiments in the circulation. III. The lumped catenary system. J. theor. Biol. 33, 491-515.

Smith, C. E. 1991. A Laguerre series approximation the the probability density of the first passage time of the Ornstein-Uhlenbeck process. In Noise in Physical Systems and $1 / f$ Fluctuations Musha, T., S. Sato, and M. Yamamoto Eds. Tokyo: Ohmsha.

Tiku, M. L., W. Y. Tan and Y. Balakrishnan. 1986. Robust Inference. New York: Dekker.

Waterhouse, C. and J. Keilson. 1972. Transfer times across the human body. Bull. Math. Biophys. 34, 33-44.

Weiss, M. 1982. Moments of physiological transit times distributions and the time course of drug disposition in the body. J. Math. Biol. 15, 305-318.

Weiss, M. 1984. A note on the role of generalized inverse Gaussian distribution of circulatory transit times in pharmacokinetics. J. Math. Biol. 20, 95-102.

Weiss, M. 1986. Generalizations in linear pharmacokinetics using properties of certain classes of residence time distributions. I. Log-convex drug disposition curves. $J$. Pharmacokin. Biopharmac. 14, 635-657.

Weiss, M. 1991. Residence time distributions in pharmacokinetics: Behavioral and structural models. In Advanced Methods of Pharmacokinetics and Pharmacodynamics System Analysis D'Argenio Ed. New York: Plenum Press.

Wise, M. E. 1985. Negative power functions of time in pharmacokinetics and their implications. J. Pharmacokin. Biopharmac. 13, 309-346.

Wise, M. E. and G. J. J. M. Borsboom. 1989. Two exceptional sets of physiological clearance curves and their mathematical form: test cases? Bull. Math. Biol. 51, 579596. 
Text to Figures:

Fig. 1. The role of the parameters $A$ and $B$ in influencing the shape of $g(x)$ given by (3.3) for $\alpha=1.5, A= \pm 0.5, B= \pm 0.5$.

Fig. 2. The L-series approximation for the known density for an exponential cycle-time distribution and Poisson elimination. The exact residence-time density has been computed by using (5.1) with parameters $\beta=1, \nu=0.5,1$ and 3 . The corresponding Lseries approximation curves are very close to these densities and are characterized by being equal zero at time zero.

Fig. 3. L-series approximations of residence-time density for a gamma distributed cycle time with parameters $\beta=1 ; \alpha=2,1,0.5$ and 0.1 ; and Poisson elimination with corresponding parameter $\nu=0.5,1,2$ and 10. For $\nu \rightarrow \infty$ and $\alpha \rightarrow 0_{+}$, such that $\nu \alpha \rightarrow \psi$, the residence-time density is a gamma density given by (5.5). It is illustrated here with $\psi=1$ and thus an exponential distribution of the residence time is achieved as a limit. Note that for $\nu=10$ and $\alpha=0.1$, (1) the limit is almost achieved, (2) L-series approximation is close to the limiting curve.

Fig. 4. The L-series approximation to the residence-time density assuming that the cycle time has an IG distribution (5.7) with $a=0.25$ and $b=1$. (a). Geometric elimination with $p=0.1,0.3,0.5,0.7,0.9$ and 1 . The hypothetical case $p=1$, the residence time and the cycle coincide, serves again to check the method. Both the theoretical curve, as well as its L-series approximation are shown. For small $p$, the limiting behavior is exponential and L-series approximation suggests it. (b). Poisson elimination with $\nu=1 / p-1, p=0.1,0.3,0.5,0.7$ and 0.9 , the values of $p$ rather than $\nu$ are given on the figure to facilitate comparison with (a).

Fig. 5. Plot of $\sqrt{\beta_{1}}$ versus $\mathrm{CV}$ of the residence time for the model with an IG cycle time. The four curves represent cycle time CV's of $0.5,0.8,1.2$ and 1.5 . (a). Geometric elimination. In these four cases, $p$ changes continuously from one (the cycle time and residence time coincides and are both $\mathrm{IG}$ ) to zero (the residence time has exponential distribution characterized by $\mathrm{CV}=1$ and $\sqrt{\beta_{1}}=2$ ). (b). Poisson elimination. In these four cases, $\nu$ changes continuously from zero (the cycle time and residence time coincides and are both IG) to infinity (the residence time is characterized by $\mathrm{CV} \rightarrow 0_{+}$ and $\left.\sqrt{\beta_{1}} \rightarrow 0_{+}\right)$.

Fig. 6. Plots of excess $\beta_{2}-3$ versus skewness $\beta_{1}$, for the model with IG cycle time and Poisson elimination. Four cases are depicted in which $\nu$ continuously changes from zero (the cycle time and residence time coincides and are both IG) to infinity (see Fig. 5b.). 


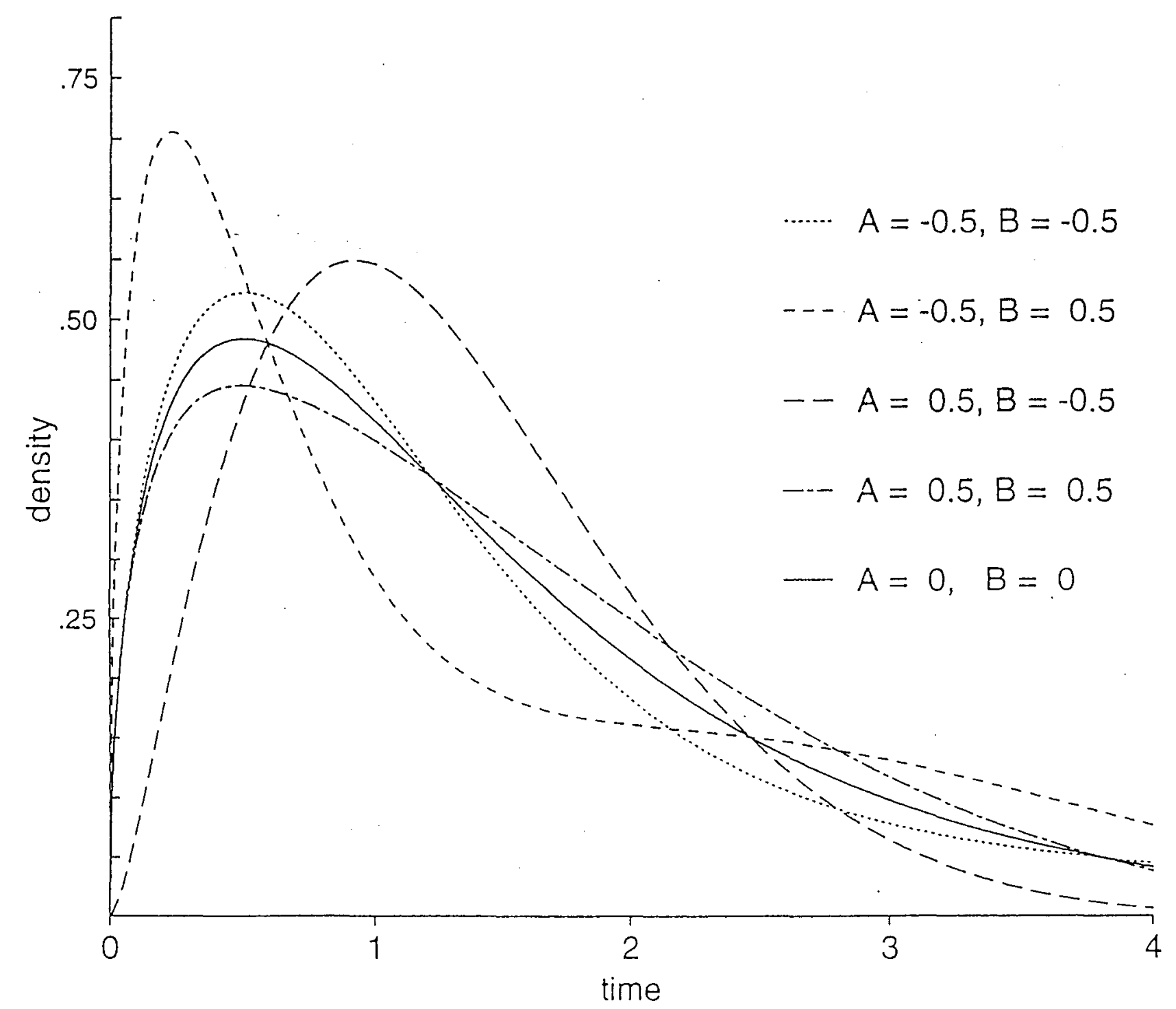

Fig. 1. 


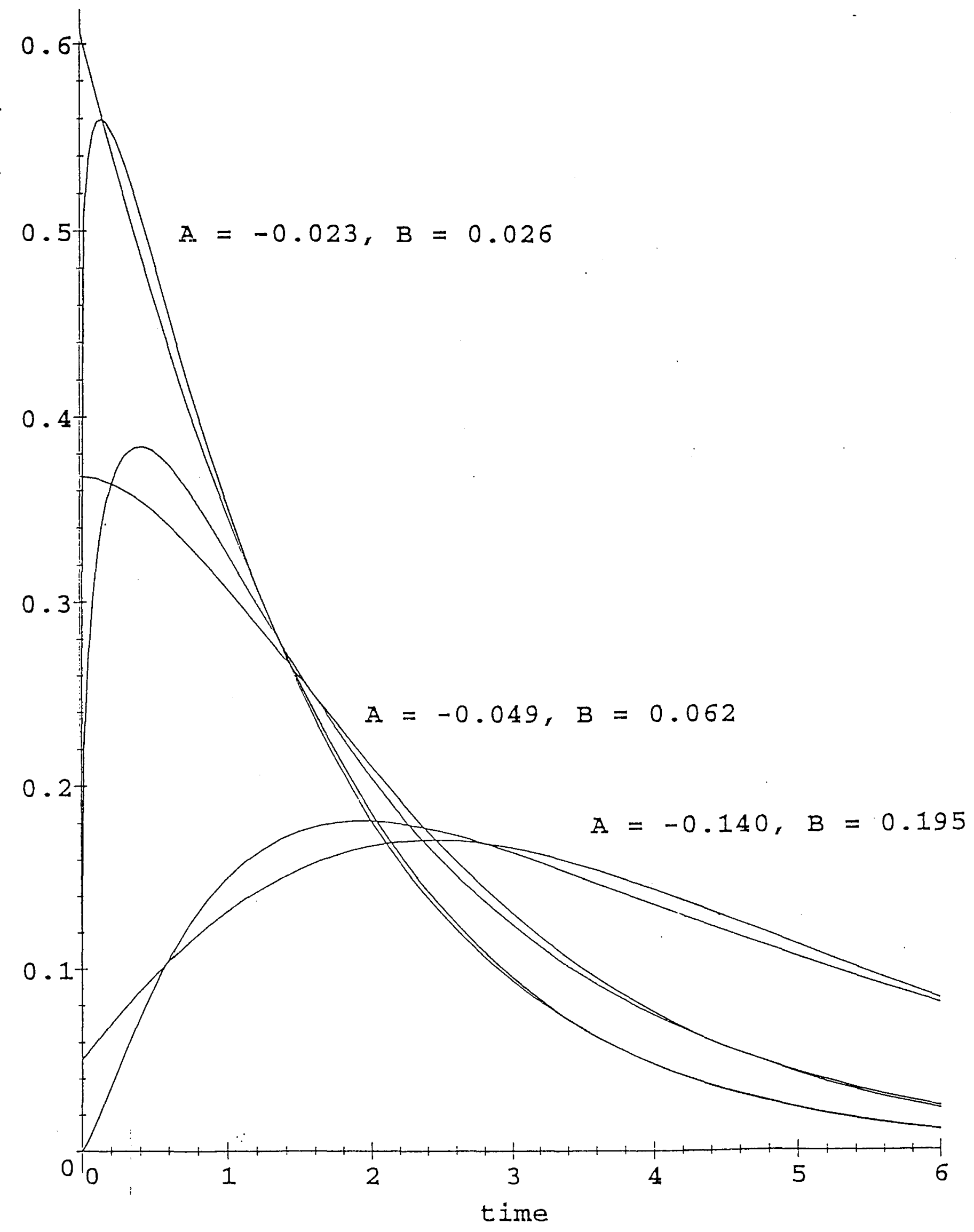

Fig. 2. 


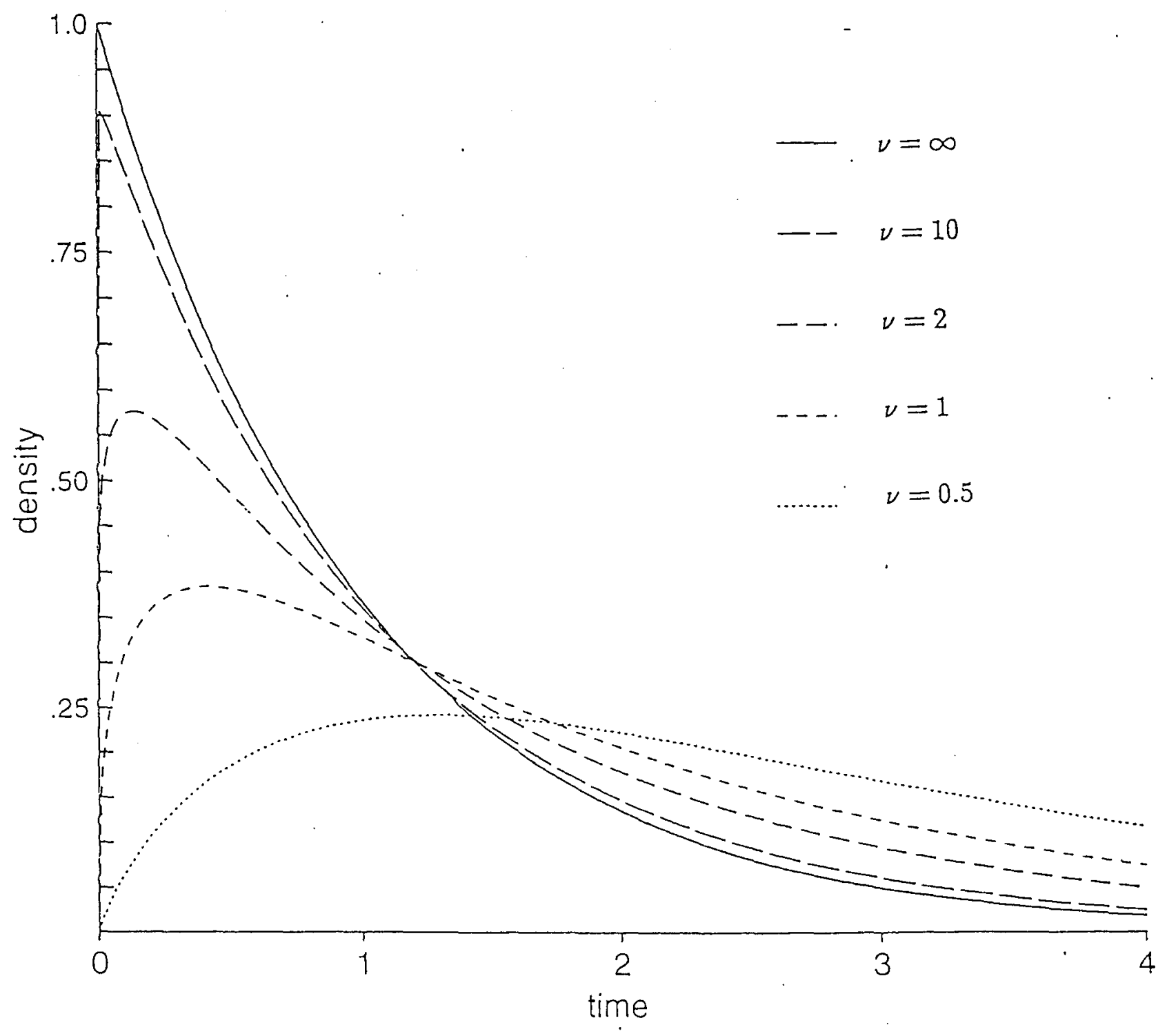

Fig. 3. 


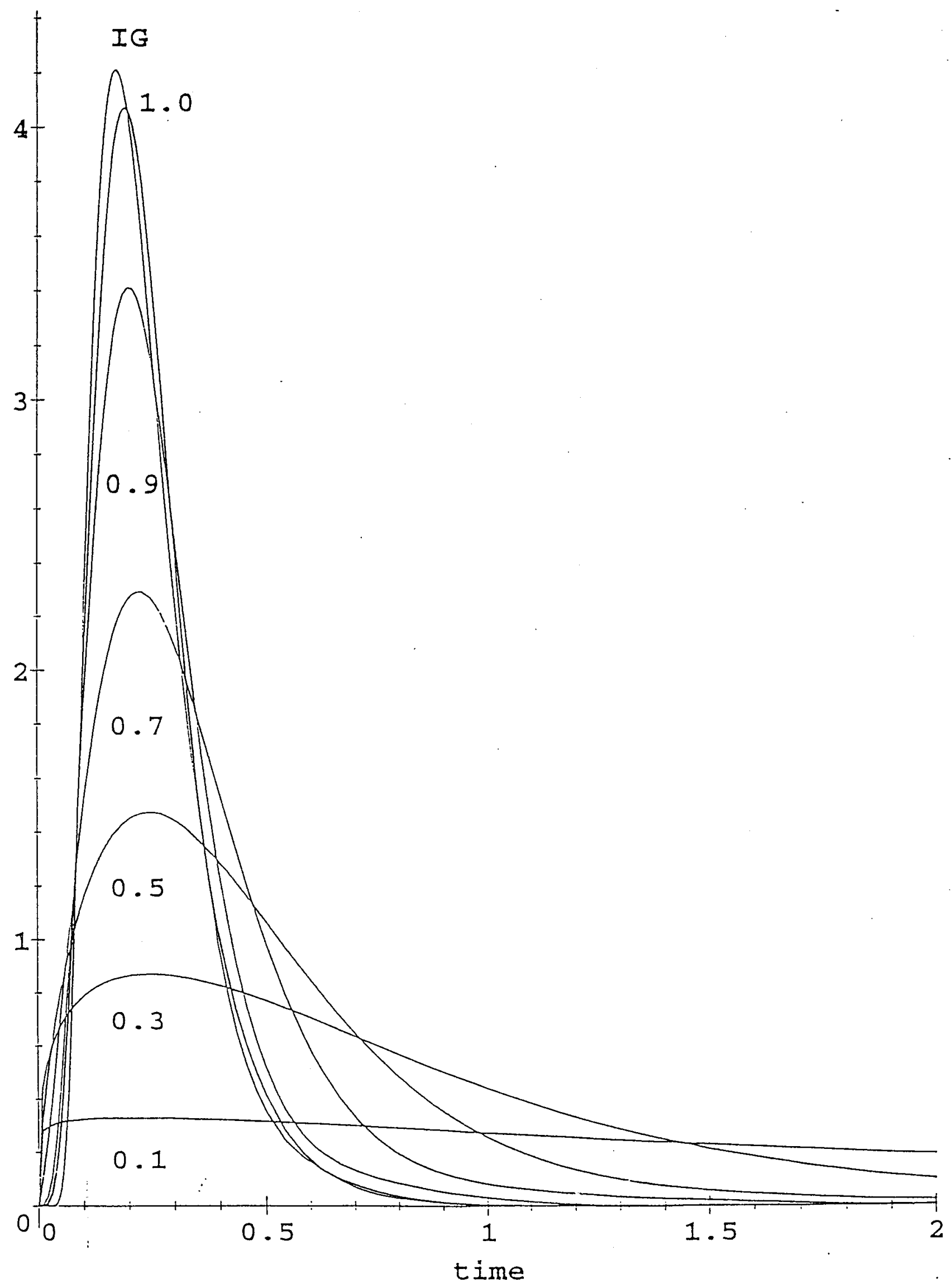

Fig. 4a. 


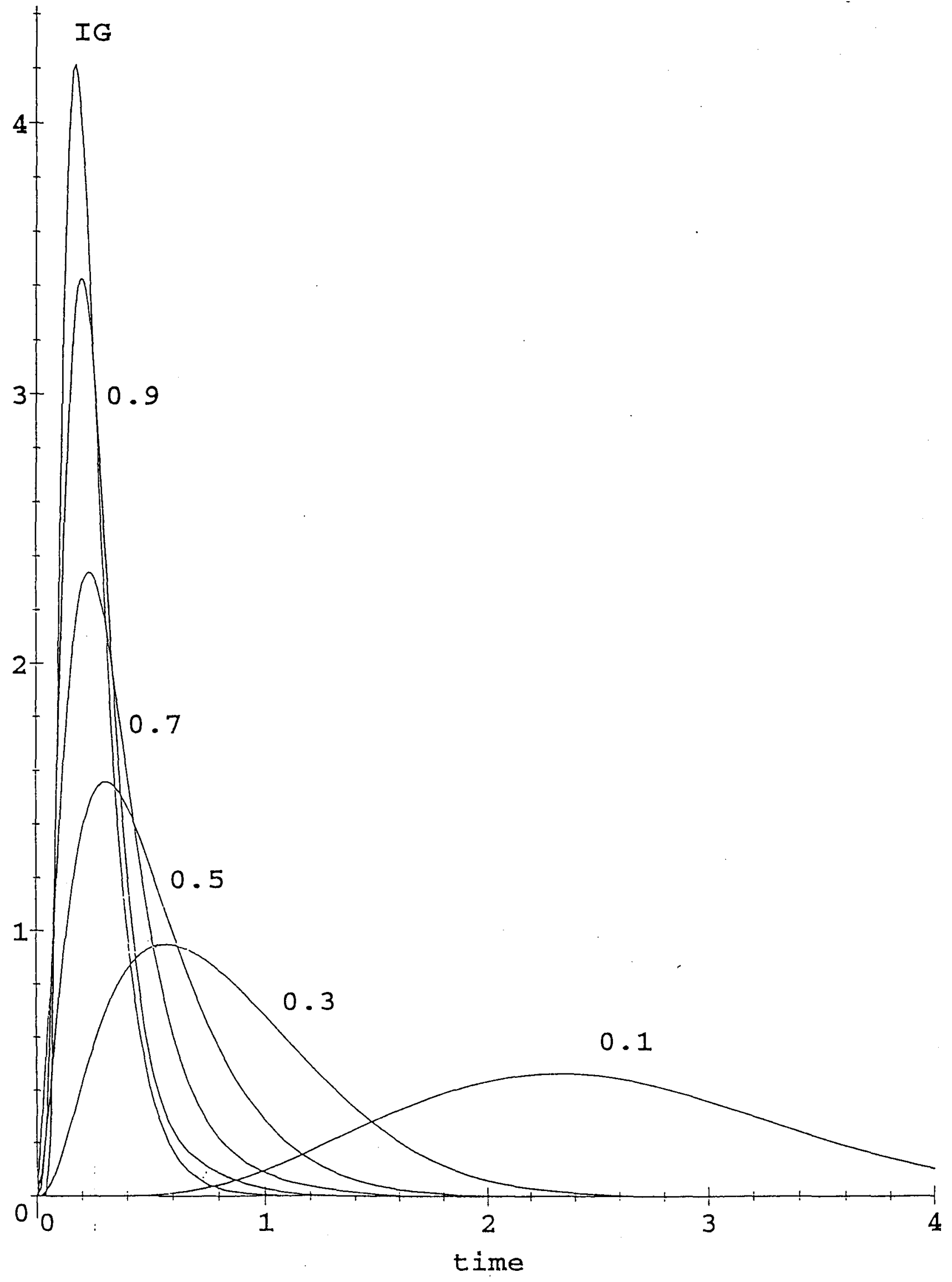

Fig. 4b. 


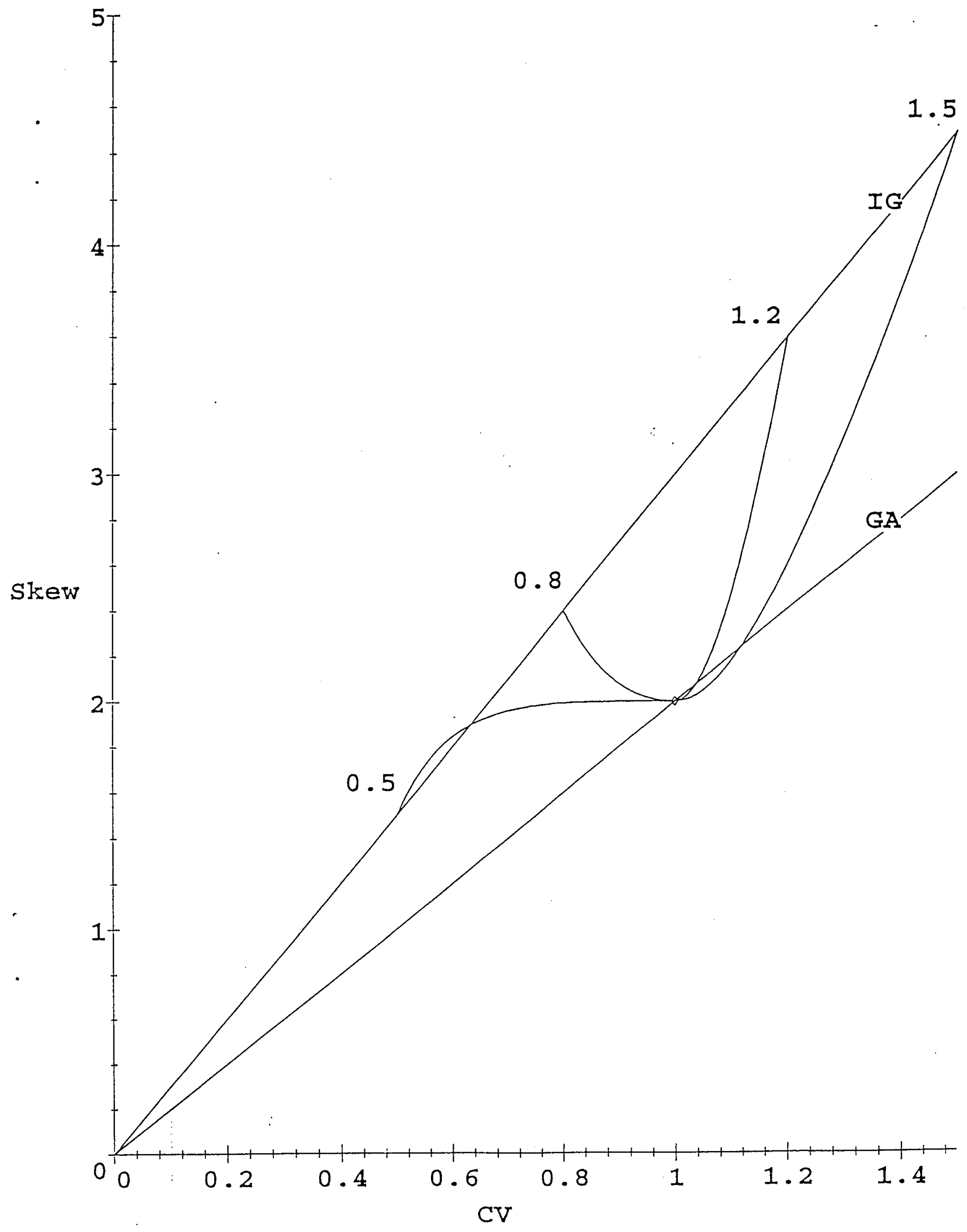

Fig. 5a. 


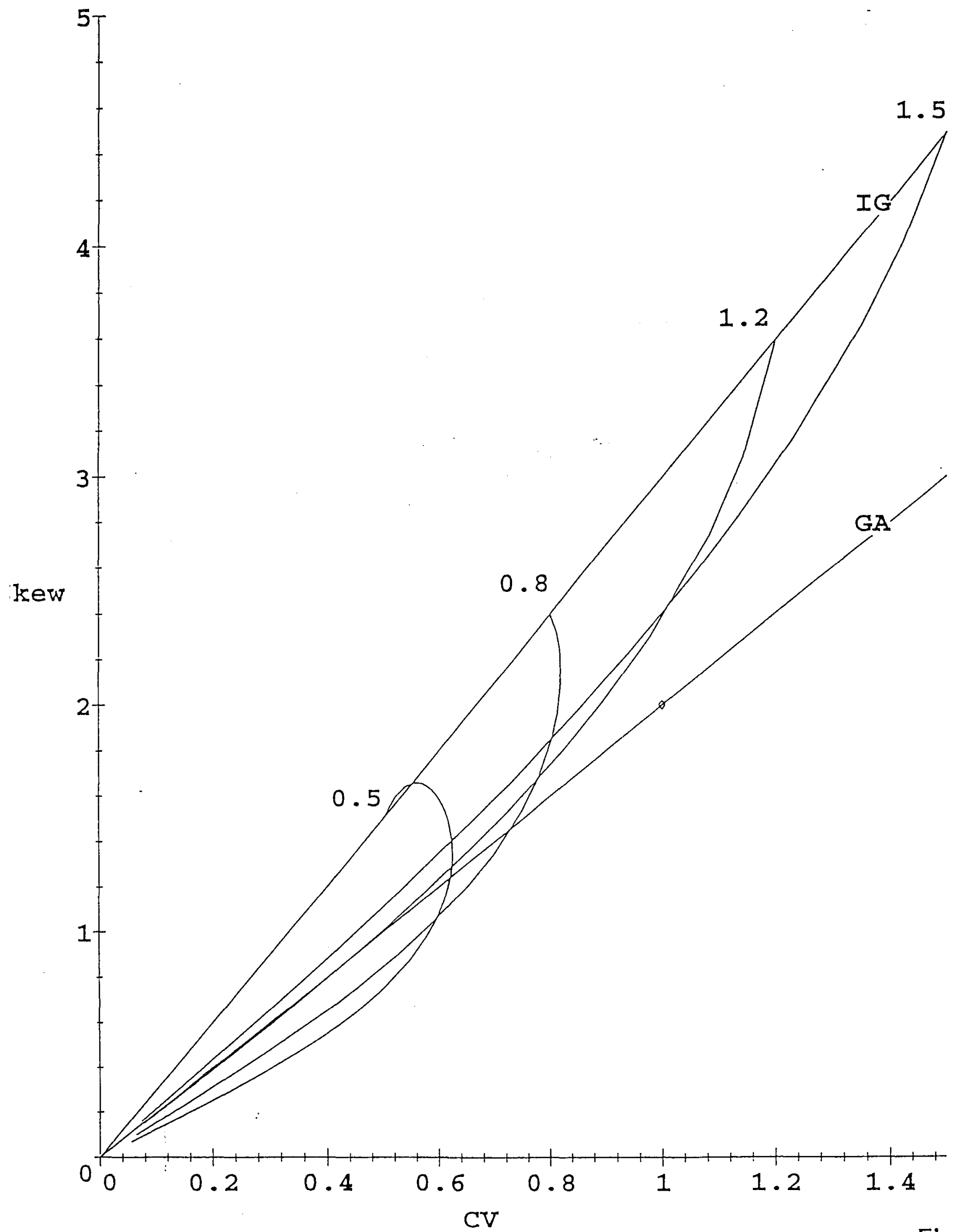

Fig. 5b. 


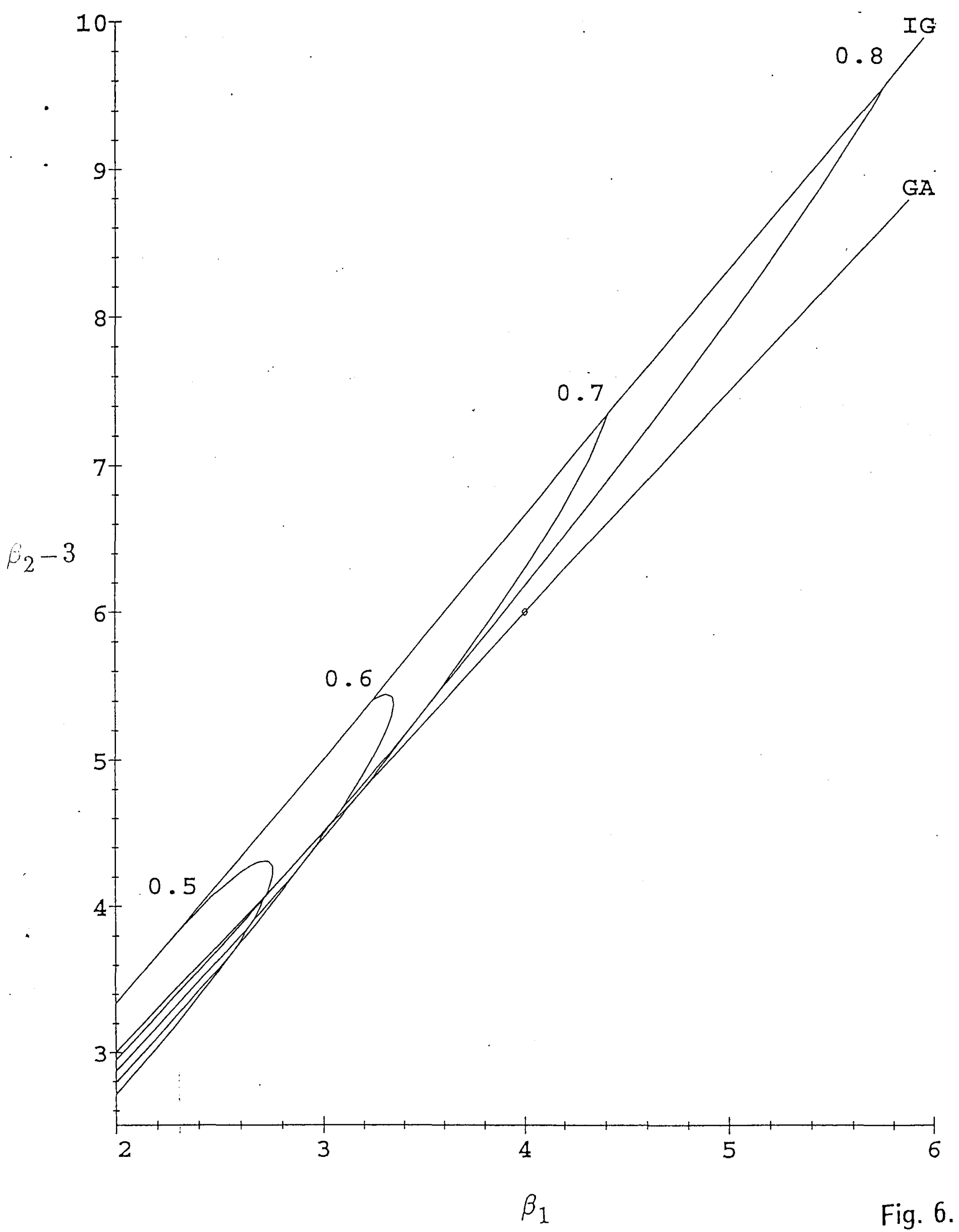

